\title{
Oral appliance treatment outcome can be predicted by continuous positive airway pressure in moderate to severe obstructive sleep apnea
}

\author{
Anders Storesund $^{1}$ (i) • Anders Johansson ${ }^{1,2}$ • Bjørn Bjorvatn ${ }^{1,3} \cdot$ Sverre Lehmann $^{1,4}$
}

Received: 13 March 2017 /Revised: 29 September 2017 / Accepted: 9 October 2017 / Published online: 24 October 2017

(C) The Author(s) 2017. This article is an open access publication

\begin{abstract}
Background Studies show that the therapeutic CPAP pressure is associated with oral appliance (OA) treatment outcome in obstructive sleep apnea (OSA) patients. However, these studies included either CPAP adherent patients using fixed pressure devices, or partly CPAP non-adherent patients using fixed pressure or auto-adjusting (auto-CPAP) devices. In many countries, auto-CPAP is predominately used, and only those non-adherent to therapy need a change to OA. Therefore, studies examining the relationship between CPAP pressures and $\mathrm{OA}$ treatment outcome should focus on patients non-adherent to auto-CPAP.

Purpose The purpose of this paper is to assess if CPAP pressures predicted OA treatment outcome in patients nonadherent to auto-CPAP therapy.

Methods The OA treatment responders and non-responders were defined by two success criteria ((1) AHI $<5$; (2) $5 \leq \mathrm{AHI}<10$ and $>50 \%$ AHI reduction). Logistic regression analyses were performed for CPAP pressures and baseline variables. ROC curve analyses were used to identify CPAP pressure cutoff values, alone and combined with other explanatory variables, predicting the $\mathrm{OA}$ treatment outcome.
\end{abstract}

Anders Storesund

anders.kjellevold.storesund@helse-bergen.no

1 Department of Thoracic Medicine, Center for Sleep Medicine, Haukeland University Hospital, Bergen, Norway

2 Department of Clinical Dentistry-Prosthodontics, Faculty of Medicine and Dentistry, University of Bergen, Bergen, Norway

3 Department of Global Public Health and Primary Care, University of Bergen, Bergen, Norway

4 Department of Clinical Science, University of Bergen, Bergen, Norway
Results Eighty-seven patients with moderate or severe OSA were included. Maximum CPAP pressures (CPAPmax) were higher in non-responders by both criteria and were, together with baseline $\mathrm{AHI}$, associated with the OA treatment outcome in multivariate regression analyses. $\mathrm{ROC}$ curves identified an optimal CPAPmax cutoff of $12 \mathrm{~cm} \mathrm{H}_{2} \mathrm{O}$, corresponding to a positive predictive value (PPV) of 0.85 in predicting nonresponse using criterion 1 . A prediction model combining CPAPmax $>12$ and baseline AHI $\geq 30$ had a PPV of 1.0 for non-response by both criteria.

Conclusions Maximum CPAP pressure was a moderate predictor of OA treatment outcome, but combined with baseline AHI, the ability to identify OA non-responders was high.

Keywords Obstructive sleep apnea · Oral appliances · Continuous positive airway pressure $\cdot$ Apnea-hypopnea index $\cdot$ Treatment outcome $\cdot$ Prediction

\section{Introduction}

Obstructive sleep apnea (OSA) is a common condition in which the upper airways intermittently collapse during sleep, causing partial or total cessation of airflow. This results in fragmentation of sleep and oxygen desaturations and is associated with neurocognitive impairment and an increased risk of cardiovascular disease and all-cause mortality $[1,2]$. The prevalence of OSA in a large Norwegian adult population has been estimated to $8 \%$ for AHI $\geq 15$ [3]. Such findings imply that OSA could have an effect on public health. Continuous positive airway pressure (CPAP) has been considered as the gold standard treatment, as it alleviates obstruction, and is shown to reduce the negative consequences of OSA [4]. However, the patient adherence is often poor, reducing the efficacy of CPAP [5]. Oral appliance (OA) is a device that 
maintains the upper airways patency preventing collapse and is a treatment alternative to CPAP in mild to moderate OSA, or when CPAP treatment fails [6]. OA is in general inferior in terms of reducing OSA parameters based on polysomnography [7], and around one third of these patients will have minimal improvement [8]. Nevertheless, OA treatment might be as effective as CPAP in mild to moderate sleep apnea, if titrated sufficiently [9]. Furthermore, OA adherence may be higher than for CPAP suggesting similar health outcomes on a group level for the two treatment modalities [10]. Thus, predictors of the OA treatment response are desired, preferably using unsophisticated measurements that are available as part of the daily clinical practice.

Various clinical and polysomnographic characteristics, like younger age, female gender, less obesity, position dependent OSA, and lower AHI, have been reported to correlate with OA treatment success [11-13]. The results, however, may be difficult to implement in a clinical setting, as they are not always consistent $[12,13]$, and because they rarely propose cutoff levels that precisely discriminate responders from non-responders, but merely lend support to the decision making when selecting therapy.

The pressure data from previous CPAP therapy is easily accessible. A therapeutic CPAP pressure $>10.5 \mathrm{~cm} \mathrm{H}_{2} \mathrm{O}$ has been associated with $\mathrm{OA}$ treatment failure in a Japanese population [14], whereas a CPAP pressure $\geq 13 \mathrm{~cm} \mathrm{H}_{2} \mathrm{O}$ predicted treatment failure in Australian patients [15]. In a Canadian study, a therapeutic CPAP pressure $\leq 9 \mathrm{~cm} \mathrm{H}_{2} \mathrm{O}$ was associated with OA treatment success [16]. The two former studies selected data from fixed pressure CPAP users with acceptable adherence, whereas the latter study included both fixed pressure and auto-CPAP users, with $50 \%$ of the subjects nontolerant to previous CPAP. In Norway, as in many other countries, the vast majority of subjects with moderate and severe OSA start with auto-adjusting CPAP machines, and only the non-adherent cases are in need of modality change to a second line treatment, such as OA.

Thus, we sought to assess the relationship between the delivered auto-CPAP pressures and the OA treatment response in a Norwegian sleep clinic population, among patients with moderate and severe OSA, all non-adherent to CPAP.

\section{Material and methods}

\section{Patients}

In this retrospective study, the patients were consecutively recruited from the Center for Sleep Medicine at the Haukeland University Hospital, Bergen, Norway, as described in a previous paper [17]. In brief, inclusion criteria were patients diagnosed with moderate or severe obstructive sleep apnea, non-adherent to CPAP therapy and subsequently treated with oral appliance. Non-adherence was defined as less than $4 \mathrm{~h}$ usage per night during a period of at least 3 months [18]. Exclusion criteria were central sleep apnea and contraindications to oral appliance therapy (e.g., poor dental status).

\section{Study protocol}

The study protocol was approved by the Regional Committees for Medical and Health Research Ethics (number 2015/2225). All subjects had a sleep study performed at baseline before starting CPAP and attended the follow-up appointment including a new sleep study using the OA. Data on body mass index (BMI) and AHI were retrieved from the patients' medical records and sleep polygraphy, retrospectively.

\section{Respiratory polygraphic evaluation}

The baseline diagnosis of OSA and follow-up investigations were performed by respiratory medicine specialists or ENT specialists at the Departments of Thoracic Medicine and Otolaryngology at Haukeland University Hospital, Bergen, Norway, supported by a medical examination that included polygraphy (Embletta ${ }^{\mathrm{TM}}$, Resmed Ltd., Australia or NOXT3®, Nox Medical, Iceland). Scoring rules were in accordance with the 2007 American Academy of Sleep Medicine manual [19].

\section{CPAP}

All patients were treated with Resmed CPAP devices in the Autoset mode. The initial pressure settings were $4-20 \mathrm{~cm}$ $\mathrm{H}_{2} \mathrm{O}$. CPAP compliance, expressed as the mean number of hours and minutes machine use every $24 \mathrm{~h}$, and delivered CPAP pressures were recorded using CPAP software (ResScan, ResMed Corp. San Diego, CA, USA). The mean value $\left(\mathrm{cm} \mathrm{H}_{2} \mathrm{O}\right)$ of the maximum CPAP pressures (CPAPmax) from each treatment session was used in the subsequent analyses. The software from ResMed auto-CPAP machines has exhibited a good diagnostic accuracy to identify residual sleep apnea, delivered pressure and mask leakage curves [20].

\section{Oral appliance}

Maxillary and mandibular impressions and an occlusal protrusive wax or silicone index using George Gauge bite fork ${ }^{\mathrm{TM}}$ were made for all subjects, with a baseline fitting index at 50 $80 \%$ of maximum protrusive capacity. The majority of patients received a titrable twin-block oral appliance while a few subjects were treated with monoblock appliances [17]. The first evaluation of subjective treatment effect was performed 4-8 weeks after starting OA. If not satisfactory, titrations were performed until positive subjective effect or until 
maximum adjustments had been done, after which an overnight polygraphy was performed.

\section{Defining treatment outcome}

Two different criteria were applied when defining successful outcome with oral appliance: criterion $1 \mathrm{AHI}<5$ and criterion $25 \leq \mathrm{AHI}<10$ and $>50 \%$ reduction from baseline.

\section{Statistical analysis}

Comparisons between the OA outcome groups were made using student's $t$ test or Mann-Whitney $U$ test for continuous variables, and chi-square test or Fisher's exact test for categorical variables. Normality was assessed using the Shapiro-Wilk test. Anthropometrical and polygraphic data associated with the OA treatment outcomes from univariate analyses were added to the delivered CPAP pressures as explanatory variables in multivariate logistic regression models. Receiver operating characteristic (ROC) curve analyses were performed to identify CPAP pressure values with the highest sensitivity and specificity related to the OA treatment outcome. Other variables associated with $\mathrm{OA}$ failure from the multivariate regression models were added to CPAP pressures in the ROC curves, to assess their combined predictive utility. $P$ values less than 0.05 were considered statistically significant. IBM SPSS (Statistics for Windows, version 23.0, IBM Corp., Armonk, NY, USA) was used for statistical analyses.

\section{Results}

During the years 2007-2013, a total of 116 patients (68\% males), predominantly Caucasians, were identified with a baseline diagnosis of moderate $(75 \%)$ or severe OSA, who had received OA treatment due to CPAP non-adherence. Among these, 29 patients (25\%) were lost to follow up for various reasons: 17 subjects had no available CPAP data, 7 did not show up for follow-up appointment, 3 died, and 2 had their follow-up elsewhere, leaving 87 patients to be included in the final analyses. Among the 87 patients, $30 \%$ had severe OSA. The patients lost to follow up did not differ from those included in this study, whose baseline and CPAP pressure characteristics are described in Table 1. The reasons for discontinuing CPAP are shown in Table 2.

The treatment success rates were $36 \%$ by criterion 1 and $64 \%$ by criterion 2 . The CPAP median usage time (hours/ night) (25-75 percentile) for all subjects was $1.2(0.5-2.2)$ and did not differ between the outcome groups by any criterion (results not shown).

The OA non-responders had significantly higher CPAPmax than responders (Table 1). Using univariate logistic regression analyses, the CPAPmax was a predictor of the OA outcome by both success criteria, with an odds ratio $[95 \% \mathrm{CI}]=1.25[1.04-1.49]$ by criterion 1 and 1.30 [1.07-1.58] by criterion 2 . When adjusted for other baseline variables in multivariate regression analyses, CPAPmax retained its ability to discriminate responders from non-responders (Table 3).

Calculating the ROC curves for CPAPmax by criterion 1 and 2 , the areas under the curve indicated moderate ability to discriminate between responders and non-responders (Fig. 1). Negative and positive predictive values, as well as likelihood ratios were calculated for different CPAP cutoff pressures (Table 4). Using criterion 1, a $12 \mathrm{~cm} \mathrm{H}_{2} \mathrm{O}$ cutoff had a positive predictive value of 0.85 in predicting non-responders, involving $39 \%$ of the study population. No such cutoff pressure was identified by criterion 2, even though a CPAPmax $>14 \mathrm{~cm}$

Table 1 Anthropometrical, polygraphic, and CPAP data for all patients and according to oral appliance treatment outcome

\begin{tabular}{|c|c|c|c|c|c|}
\hline & \multirow[t]{2}{*}{ All patients } & \multicolumn{2}{|l|}{ Criterion $1 \mathrm{AHI}<5$} & \multicolumn{2}{|c|}{ Criterion $25 \leq \mathrm{AHI}<10$ and $>50 \%$ AHI reduction } \\
\hline & & Responders & Non-responders & Responders & Non-responders \\
\hline Number & 87 & 31 & 56 & 56 & 31 \\
\hline Sex (male/female) & $59 / 28$ & $21 / 10$ & $38 / 18$ & $37 / 19$ & $22 / 9$ \\
\hline Age (years) & $56.7 \pm 11.7$ & $55.5 \pm 8.7$ & $57.4 \pm 13.1$ & $55.3 \pm 11.1$ & $59.3 \pm 12.5$ \\
\hline $\mathrm{BMI}\left(\mathrm{kg} / \mathrm{m}^{2}\right)$ & $28.6 \pm 4.2$ & $28.2 \pm 3.8$ & $28.8 \pm 4.4$ & $28.7 \pm 3.9$ & $28.5 \pm 4.7$ \\
\hline OSA, severity (moderate/severe) & $61 / 26$ & $25 / 6$ & $36 / 20$ & $44 / 12 *$ & $17 / 14$ \\
\hline AHI, baseline & $23.8(19.6-32.2)$ & $20.8(18.0-26.0)^{*}$ & $24.8(20.3-39.1)$ & $21.2(18.2-26.9)^{* *}$ & $28.3(22.4-45.5)$ \\
\hline AHI, oral appliance & $6.9(4.0-13.7)$ & $3.1(1.4-4.1)^{* *}$ & $10.9(7.1-20.0)$ & $4.9(3.0-6.8)^{* * *}$ & $18.2(13.5-30.6)$ \\
\hline AHI, CPAP & $3.8(1.9-7.7)$ & $3.1(0.8-9.3)$ & $4.4(2.2-7.7)$ & $3.4(2.0-5.6)$ & $5.5(1.6-10.7)$ \\
\hline Maximum CPAP pressure, $\mathrm{cm} \mathrm{H}_{2} \mathrm{O}$ & $11.2 \pm 2.8$ & $10.1 \pm 2.9^{*}$ & $11.7 \pm 2.6$ & $10.5 \pm 2.6^{* *}$ & $12.3 \pm 2.9$ \\
\hline
\end{tabular}

Data are presented as numbers, mean \pm standard deviation or median $(25-75 \%)$

$B M I$, body mass index; $O S A$, obstructive sleep apnea; $A H I$, apnea-hypopnea index; $C P A P$, continuous positive airway pressure

${ }^{*} p<0.05$ responders versus non-responders; $* * p<0.01$ responders versus non-responders; $* * * \mathrm{p}<0.001$ responders versus non-responders 
Table 2 Reasons for discontinuation of continuous positive airway pressure (CPAP)

\begin{tabular}{ll}
\hline & No. $(\%)(n=87)$ \\
\hline Noise or discomfort of machine & $26(30)$ \\
Claustrophobia & $20(23)$ \\
Insomnia & $17(19)$ \\
Side effects (e.g., headache, sinusitis) & $12(14)$ \\
Practical issues (e.g., travelling, power supply) & $6(7)$ \\
Tears it off during sleep & $4(5)$ \\
Unknown & $2(2)$ \\
\hline
\end{tabular}

$\mathrm{H}_{2} \mathrm{O}$ correctly classified a larger proportion of the study population.

Baseline AHI had significant predictive value by both criteria (Tables 1 and 3). By adding the baseline AHI to the CPAPmax in a simple prediction model, the ROC AUC $(95 \%$ $\mathrm{CI})$ increased to $0.70(0.59-0.81, p=0.002)$ and $0.73(0.61-$ $0.85, p<0.001$ ) by criterion 1 and 2 , respectively (Fig. 1 ).

A prediction model using a CPAPmax $>12 \mathrm{~cm} \mathrm{H}_{2} \mathrm{O}$ and baseline $\mathrm{AHI} \geq 30$ as cutoff values had a positive predictive value (PPV) of 1.0 for OA non-response both by criterion 1 and criterion 2 (Table 5).

\section{Discussion}

In this study, we sought to investigate the relationship between auto-CPAP pressures and OA treatment outcome in moderate to severe obstructive sleep apnea patients all non-adherent to CPAP treatment. Our findings indicate that the pressures delivered from auto-CPAP machines were associated with the subsequent $\mathrm{OA}$ treatment outcome. Combining the easily available maximum pressure data from the CPAP software with the AHI from the baseline respiratory polygraphy, cutoff values with high predictive values for $\mathrm{OA}$ treatment failure were identified.

The results are in accordance with the findings in the previously mentioned studies [14-16]. However, as in the
Canadian study [16], the correlation between CPAP pressures and $\mathrm{OA}$ treatment outcome was not as strong as reported in the Japanese and Australian populations [14, 15]. There might be several reasons for this.

First, we have to consider the possible effect of the differences in the pressure modes used. All patients in the present study used CPAP in auto pressure mode, whereas fixed pressure CPAP was applied for treatment in the Japanese and Australian studies. The fixed pressure level was defined either by manual in-laboratory titration [14] or the 95th percentile pressure from auto-CPAP [15] and confirmed to be therapeutic with polysomnography. In the Canadian study [16], the pressure data were retrieved from a variety of commercially available CPAP machines, mainly of auto-adjusting type, using the 90th percentile pressure. Auto-CPAP devices adjust the treatment pressure based on feedback from various patient measures (airflow, pressure fluctuations, measures of airway resistance), applying the lowest effective pressure to splint the airways open. The mode takes into account the pressure variation during sleep over a given period of time. A single-night titration, determining the fixed pressure requirements either manually or by auto-CPAP, may be affected by an inadequate sampling of sleep position and quality [21]. Studies comparing the different modes in respect to pressure levels have shown that the therapeutic pressures are lower using an automatic mode [22]. The therapeutic pressure requirements also vary with the CPAP manufacturer and device algorithm [21]. Consequently, the differences in the pressures reported in various publications may depend on the choice of CPAP study design.

Second, in the Australian and Japanese studies [14, 15], the inclusion criteria comprised adherence to CPAP, indicating that the participants were able to sleep most of the night during treatment. Thus, breathing cessations demanding higher therapeutic pressures will occur more frequently in the CPAP adherent, sleeping patient compared to the partially awake patient struggling with mask and machine acceptance. In our study, all patients were non-adherent to CPAP therapy. However, this does not necessarily mean that they were truly non-compliant. The reasons for discontinuing CPAP treatment were diverse, some of which might relate to high pressure
Table 3 Oral appliance treatment outcome predicted by maximum CPAP pressure $\left(\mathrm{cm} \mathrm{H}_{2} \mathrm{O}\right)$, anthropometrical data, and polygraphic variables. Multivariate logistic regression analyses

\begin{tabular}{|c|c|c|c|c|}
\hline & \multicolumn{2}{|c|}{ Criterion 1 AHI $<5$} & \multicolumn{2}{|c|}{$\begin{array}{l}\text { Criterion } 25 \leq \mathrm{AHI}<10 \text { and }>50 \% \mathrm{AH} \\
\text { reduction }\end{array}$} \\
\hline & $p$ & Odds ratio $(95 \% \mathrm{CI})$ & $p$ & Odds ratio $(95 \% \mathrm{CI})$ \\
\hline Sex & 0.74 & $0.83(0.28-2.48)$ & 0.86 & $1.11(0.34-3.64)$ \\
\hline Age & 0.35 & $1.02(0.98-1.07)$ & 0.05 & $1.05(1.00-1.10)$ \\
\hline BMI & 0.43 & $1.05(0.93-1.18)$ & 0.99 & $1.00(0.88-1.13)$ \\
\hline AHI, baseline & 0.02 & $1.07(1.01-1.13)$ & 0.001 & $1.09(1.04-1.14)$ \\
\hline CPAP maximum pressure & 0.02 & $1.27(1.05-1.55)$ & 0.006 & $1.38(1.10-1.74)$ \\
\hline
\end{tabular}

$B M I$, body mass index; $A H I$, apnea-hypopnea index; CPAP, continuous positive airway pressure 


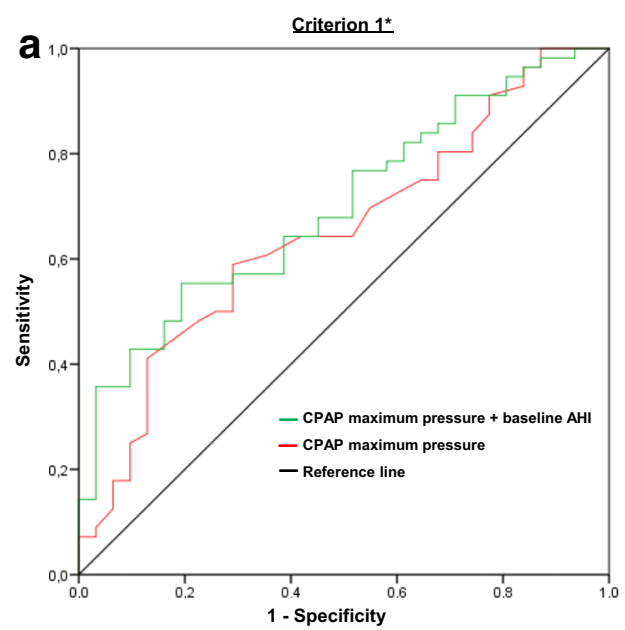

Fig. 1 Receiver operating characteristics (ROC) curves for CPAP maximum pressure alone and in combination with baseline AHI, using oral appliance $(\mathrm{OA})$ treatment success criterion $1^{*}$ and criterion $2^{* *}$ as outcome variables. a Criterion $1(\mathrm{AHI}<5)$ : The area under the curve (AUC) for CPAP maximum pressure was $0.65(0.53-0.77, p=0.02)$ versus 0.70 $(0.59-0.81, p=0.002)$ for a prediction model combining CPAP

levels, whereas others could be "pressure independent," e.g., lack of motivation. To receive reimbursement for OA therapy in Norway, non-adherence to previous CPAP therapy is required, which might contribute to a higher fraction of unmotivated CPAP users, using the machine for only a short period of time mostly being awake. One might speculate how this would affect the CPAP pressures recorded, most likely reducing the pressure variations.

Third, the two studies $[14,15]$ also included patients with mild sleep apnea in addition to moderate and severe disease, as opposed to our study, which included only patients with moderate or severe sleep apnea. Still, the baseline AHI was higher in both studies, indicating a wider range of AHI, with more patients in each end of the severity scale. An elevated $\mathrm{AHI}$ at baseline is associated with an increased risk of OA treatment failure [23]. Baseline AHI has also been included

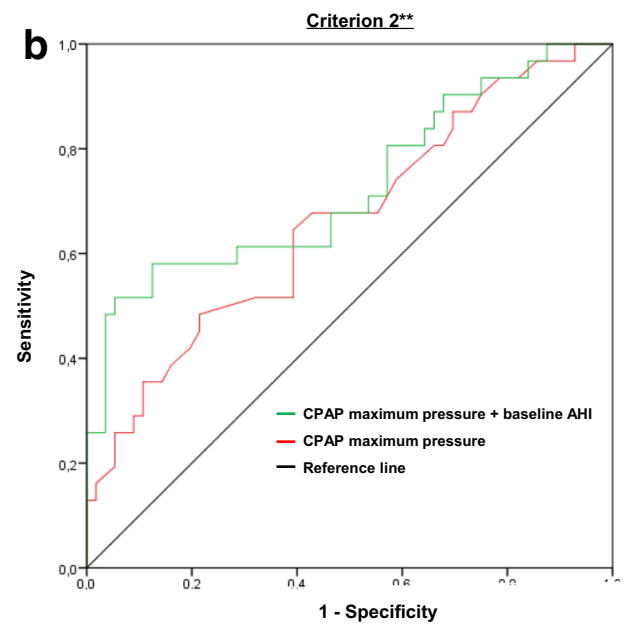

maximum pressure with baseline AHI. b Criterion $2(5 \leq \mathrm{AHI}<10$ and $>50 \%$ reduction from baseline): The area under the curve (AUC) for CPAP maximum pressure was $0.66(0.54-0.78, p=0.013)$ versus 0.73 $(0.61-0.85, p<0.001)$ for a prediction model combining CPAP maximum pressure with baseline AHI

in mathematical equations to predict the CPAP pressure needed to alleviate airway obstruction; the higher the baseline AHI, the higher the therapeutic CPAP pressure [24]. Consequently, the pressure difference observed between responders and nonresponders could be more pronounced in the referred studies than in our population.

The study populations also differ in other ways. The Japanese population had a lower BMI and included only males [14], whereas the Australian population was younger [15], compared to the present study. In the Canadian study [16], the patients were in many respects similar to ours. Accordingly, different patient selection might explain the moderate result variation between studies examining the relationship concerning CPAP pressures and OA treatment efficacy.

Sleep apnea is a heterogeneous disorder with several phenotypes related to anatomical and non-anatomical factors [25,

Table 4 The ability of different maximum CPAP pressure levels to discriminate oral appliance treatment responders from non-responders

\begin{tabular}{|c|c|c|c|c|c|c|c|c|c|c|}
\hline \multirow{3}{*}{$\begin{array}{l}\text { AUC }(95 \% \mathrm{CI}) \\
\text { Cutoff, } \mathrm{cm} \mathrm{H}_{2} \mathrm{O}\end{array}$} & \multicolumn{5}{|c|}{ Criterion $1 \mathrm{AHI}<5$} & \multicolumn{5}{|c|}{ Criterion $25 \leq \mathrm{AHI}<10$ and $>50 \%$ AHI reduction } \\
\hline & \multicolumn{5}{|c|}{$0.65(0.53-0.77)^{*}$} & \multicolumn{5}{|c|}{$0.66(0.54-0.78)^{*}$} \\
\hline & $>10$ & $>11$ & $>12$ & $>13$ & $>14$ & $>10$ & $>11$ & $>12$ & $>13$ & $>14$ \\
\hline Sensitivity & 0.75 & 0.64 & 0.41 & 0.23 & 0.16 & 0.81 & 0.68 & 0.48 & 0.32 & 0.26 \\
\hline Specificity & 0.32 & 0.58 & 0.87 & 0.90 & 0.94 & 0.32 & 0.50 & 0.79 & 0.89 & 0.95 \\
\hline PPV & 0.67 & 0.74 & 0.85 & 0.82 & 0.82 & 0.40 & 0.43 & 0.56 & 0.63 & 0.73 \\
\hline NPV & 0.42 & 0.47 & 0.45 & 0.40 & 0.38 & 0.75 & 0.74 & 0.73 & 0.70 & 0.70 \\
\hline LR+ & 1.11 & 1.53 & 3.18 & 2.58 & 2.49 & 1.19 & 1.35 & 2.26 & 3.01 & 4.82 \\
\hline LR- & 0.78 & 0.62 & 0.68 & 0.83 & 0.90 & 0.60 & 0.65 & 0.66 & 0.76 & 0.78 \\
\hline$\%$ correctly classified & 60.0 & 62.1 & 57.5 & 48.3 & 43.7 & 49.4 & 56.3 & 68.9 & 68.9 & 70.1 \\
\hline
\end{tabular}

$A U C$, Area under curve; $C I$, confidence interval; $P P V$, positive predictive value; $N P V$, negative predictive value; $L R+$, positive likelihood ratio; $L R$-, negative likelihood ratio; $* p<0.05$ 
Table 5 Positive predictive values for OA treatment failure combining maximum CPAP pressure and baseline $\mathrm{AHI}$ by criterion $1 \mathrm{AHI}<5$ and criterion 2 $5 \leq \mathrm{AHI}<10$ and $>50 \% \mathrm{AHI}$ reduction $(\mathrm{C} 1 / \mathrm{C} 2)$

\begin{tabular}{lllllll}
\hline \multicolumn{7}{c}{ AHI at baseline } \\
\cline { 3 - 7 } & & $\geq 20$ & $\geq 25$ & $\geq 30$ & $\geq 35$ & $\geq 40$ \\
\hline Maximum CPAP pressure $\left(\mathrm{H}_{2} \mathrm{O}\right)$ & $>10$ & $0.74 / 0.50$ & $0.81 / 0.62$ & $0.82 / 0.65$ & $0.92 / 0.77$ & $0.91 / 0.82$ \\
& $>11$ & $0.81 / 0.54$ & $0.91 / 0.73$ & $0.92 / 0.85$ & $1.00 / 0.91$ & $1.00 / 1.00$ \\
& $>12$ & $0.95 / 0.67$ & $1.00 / 0.86$ & $1.00 / 1.00$ & $1.00 / 1.00$ & $1.00 / 1.00$ \\
& $>13$ & $0.92 / 0.75$ & $1.00 / 0.89$ & $1.00 / 1.00$ & $1.00 / 1.00$ & $1.00 / 1.00$ \\
& $>14$ & $1.00 / 0.88$ & $1.00 / 1.00$ & $1.00 / 1.00$ & $1.00 / 1.00$ & $1.00 / 1.00$ \\
\hline
\end{tabular}

26]. Reliable and simple methods for identification of these phenotypes could allow for future customized therapy [26]. However, given the diverse nature of OSA and based on the findings in this and previous studies [11-13], it seems unlikely that a single clinical or polysomnographic variable is sufficient to predict OA treatment outcome for every patient. A combination of several characteristics might have better predictive ability. Such models should be clinically applicable and aim for a high percentage of correctly classified treatment outcomes. This has been addressed in a previous study, which used models based on patient characteristics and OSA phenotypes [13]. However, no reliable prediction tool was found, and the authors concluded that other prediction methods were needed. A different approach could be to develop models that reliably identify a subset of the OA non-responders, i.e., models with high predictive value for OA non-response. In that way, other effective treatment strategies for these patients would be initiated earlier, saving health care resources. In a prospective study of moderate OSA patients, a combination of Mallampati score of class 4 and BMI $>24$ had high predictive value for OA non-response [27]. Using these cutoff levels, about $30 \%$ of all non-responders were identified. This coincides with our results. A prediction model combining the maximum CPAP pressures and baseline AHI seemed to identify a phenotypic trait not responding to OA, predicting treatment failure with high accuracy. Other methods that examine the change of upper airways dimensions during mandibular advancement, either by sleep monitoring with the simultaneous use of a remotely controlled mandibular positioner or by endoscopic evaluation, have shown promising results [28, 29], but are invasive and operator dependent and time and cost consuming.

There are other definitions of CPAP adherence than the one used in this study, e.g., $\geq 4 \mathrm{~h} /$ night in $\geq 70 \%$ of nights [5]. What constitutes optimal use is not known. There seems to be a dose-response relationship between CPAP use and clinical outcome, implying that there is a lower threshold for the duration of therapy, below which the treatment effects disappear $[4,5]$. Still, some patients who would otherwise be considered as CPAP non-adherent may profit on usage of shorter duration, without the need for treatment change [18]. Thus, the level of adherence must always be compared to the clinical effect. For the majority to benefit from treatment; however, the CPAP should be used most of the time asleep [30]. A cutoff of at least $4 \mathrm{~h}$ use per night has proven valid for various outcome measures $[18,30]$.

There are limitations and strengths to our study. The data were retrieved retrospectively, making room for bias. However, the information was collected from consecutive patients, using CPAP software and medical records, i.e., reliable sources. The Center for Sleep Medicine in the present study serves as a single regional center, which in addition to diagnosing OSA, also includes initiation of therapy and subsequent follow-up of all patients, even if they initially were evaluated by private health care providers. Thus, the patient assortment should be representative for the diagnosed OSA population. In Norway, CPAP is still the first line of treatment for all severity levels of OSA, and patients get their OA expenses reimbursed only if they fail CPAP therapy. These factors reduce the risk of selection bias.

In conclusion, this study shows that high CPAP maximum pressures are associated with subsequent $\mathrm{OA}$ treatment failure in non-compliant auto-CPAP users suffering from moderate to severe OSA. The predictive ability was further improved when pressure was combined with the baseline AHI, reflecting disease severity. A prediction model using a combination of CPAP maximum pressure $>12 \mathrm{~cm} \mathrm{H}_{2} \mathrm{O}$ and a baseline AHI $\geq 30$ as cutoff values had very high predictive values in identifying OA non-responders, but needs prospective validation.

Funding This study was partly funded by the Norwegian Competence Center for Sleep Disorders and by Chiesi Pharma AB. The funders had no role in study design, data collection and analysis, decision to publish, or preparation of the manuscript.

\section{Compliance with ethical standards}

Conflict of interest The authors declare that they have no conflict of interest.

Ethical approval All procedures performed in studies involving human participants were in accordance with the ethical standards of the institutional and/or national research committee and with the 1964 Helsinki Declaration and its later amendments or comparable ethical standards. 
Informed consent For this type of study formal consent is not required. Open Access This article is distributed under the terms of the Creative Commons Attribution 4.0 International License (http:// creativecommons.org/licenses/by/4.0/), which permits unrestricted use, distribution, and reproduction in any medium, provided you give appropriate credit to the original author(s) and the source, provide a link to the Creative Commons license, and indicate if changes were made.

\section{References}

1. Gottlieb DJ, Yenokyan G, Newman AB, O'Connor GT, Punjabi NM, Quan SF, Redline S, Resnick HE, Tong EK, Diener-West M, Shahar E (2010) Prospective study of obstructive sleep apnea and incident coronary heart disease and heart failure: the sleep heart health study. Circulation 122 (4):352360. https://doi.org/10.1161/CIRCULATIONAHA.109.901801

2. Marshall NS, Wong KK, Cullen SR, Knuiman MW, Grunstein RR (2014) Sleep apnea and 20-year follow-up for all-cause mortality, stroke, and cancer incidence and mortality in the Busselton Health Study cohort. J Clin Sleep Med: JCSM : Off Publ Am Acad Sleep Med 10(4):355-362. https://doi.org/10.5664/jcsm.3600

3. Hrubos-Strom H, Randby A, Namtvedt SK, Kristiansen HA, Einvik G, Benth J, Somers VK, Nordhus IH, Russell MB, Dammen T, Omland T, Kvaerner KJ (2011) A Norwegian population-based study on the risk and prevalence of obstructive sleep apnea. The Akershus Sleep Apnea Project (ASAP). J Sleep Res 20(1 Pt 2):162-170. https://doi.org/10.1111/j.1365-2869.2010.00861.x

4. Antic NA, Catcheside P, Buchan C, Hensley M, Naughton MT, Rowland S, Williamson B, Windler S, McEvoy RD (2011) The effect of CPAP in normalizing daytime sleepiness, quality of life, and neurocognitive function in patients with moderate to severe OSA. Sleep 34(1):111-119

5. Kribbs NB, Pack AI, Kline LR, Smith PL, Schwartz AR, Schubert NM, Redline S, Henry JN, Getsy JE, Dinges DF (1993) Objective measurement of patterns of nasal CPAP use by patients with obstructive sleep apnea. Am Rev Respir Dis 147(4):887-895. https://doi.org/10.1164/ajrccm/147.4.887

6. Ramar K, Dort LC, Katz SG, Lettieri CJ, Harrod CG, Thomas SM, Chervin RD (2015) Clinical practice guideline for the treatment of obstructive sleep apnea and snoring with oral appliance therapy: an update for 2015. J Clin Sleep Med: JCSM : Off Publ Am Acad Sleep Med 11(7):773-827. https://doi.org/10.5664/jcsm.4858

7. Gagnadoux F, Fleury B, Vielle B, Petelle B, Meslier N, N"Guyen XL, Trzepizur W, Racineux JL (2009) Titrated mandibular advancement versus positive airway pressure for sleep apnoea. Eur Respir J 34(4):914-920. https://doi.org/10.1183/09031936. 00148208

8. Sutherland K, Vanderveken OM, Tsuda H, Marklund M, Gagnadoux F, Kushida CA, Cistulli PA (2014) Oral appliance treatment for obstructive sleep apnea: an update. J Clin Sleep Med: JCSM : Off Publ Am Acad Sleep Med 10(2):215-227. https://doi.org/10.5664/jcsm.3460

9. Doff MH, Hoekema A, Wijkstra PJ, van der Hoeven JH, Huddleston Slater JJ, de Bont LG, Stegenga B (2013) Oral appliance versus continuous positive airway pressure in obstructive sleep apnea syndrome: a 2-year follow-up. Sleep 36(9):12891296. https://doi.org/10.5665/sleep.2948

10. Phillips CL, Grunstein RR, Darendeliler MA, Mihailidou AS, Srinivasan VK, Yee BJ, Marks GB, Cistulli PA (2013) Health outcomes of continuous positive airway pressure versus oral appliance treatment for obstructive sleep apnea: a randomized controlled trial. Am J Respir Crit Care Med 187(8):879-887. https://doi.org/10.1164/rccm.201212-2223OC

11. Marklund M, Stenlund H, Franklin KA (2004) Mandibular advancement devices in 630 men and women with obstructive sleep apnea and snoring: tolerability and predictors of treatment success. Chest 125(4):1270-1278

12. Holley AB, Lettieri CJ, Shah AA (2011) Efficacy of an adjustable oral appliance and comparison with continuous positive airway pressure for the treatment of obstructive sleep apnea syndrome. Chest 140(6):1511-1516. https://doi.org/10.1378/chest.10-2851

13. Sutherland K, Takaya H, Qian J, Petocz P, Ng AT, Cistulli PA (2015) Oral appliance treatment response and polysomnographic phenotypes of obstructive sleep apnea. J Clin Sleep Med: JCSM : Off Publ Am Acad Sleep Med 11(8):861-868. https://doi.org/10.5664/jcsm.4934

14. Tsuiki S, Kobayashi M, Namba K, Oka Y, Komada Y, Kagimura T, Inoue Y (2010) Optimal positive airway pressure predicts oral appliance response to sleep apnoea. Eur Respir J 35(5):1098-1105. https://doi.org/10.1183/09031936.00121608

15. Sutherland K, Phillips CL, Davies A, Srinivasan VK, Dalci O, Yee BJ, Darendeliler MA, Grunstein RR, Cistulli PA (2014) CPAP pressure for prediction of oral appliance treatment response in obstructive sleep apnea. J Clin Sleep Med: JCSM : Off Publ Am Acad Sleep Med 10(9):943-949. https://doi.org/10.5664/jcsm.4020

16. Dort LC, Savard N, Dort E, Dort M, Dort J (2016) Does CPAP pressure predict treatment outcome with oral appliances? J Dent Sleep Med 03(04):113-117

17. Gjerde K, Lehmann S, Berge ME, Johansson AK, Johansson A (2016) Oral appliance treatment in moderate and severe obstructive sleep apnoea patients non-adherent to CPAP. J Oral Rehabil 43(4): 249-258. https://doi.org/10.1111/joor.12376

18. Weaver TE, Maislin G, Dinges DF, Bloxham T, George CF, Greenberg H, Kader G, Mahowald M, Younger J, Pack AI (2007) Relationship between hours of CPAP use and achieving normal levels of sleepiness and daily functioning. Sleep 30(6):711-719

19. Berry RB, Budhiraja R, Gottlieb DJ, Gozal D, Iber C, Kapur VK, Marcus CL, Mehra R, Parthasarathy S, Quan SF, Redline S, Strohl KP, Davidson Ward SL, Tangredi MM, American Academy of Sleep M (2012) Rules for scoring respiratory events in sleep: update of the 2007 AASM manual for the scoring of sleep and associated events. Deliberations of the sleep apnea definitions task force of the American Academy of Sleep Medicine Journal of clinical sleep medicine : JCSM : official publication of the American Academy of Sleep Medicine 8 (5):597-619. doi:https://doi.org/10.5664/jcsm.2172

20. Nigro CA, Gonzalez S, Arce A, Aragone MR, Nigro L (2015) Accuracy of a novel auto-CPAP device to evaluate the residual apnea-hypopnea index in patients with obstructive sleep apnea. Sleep Breath 19(2):569-578. https://doi.org/10.1007/s11325-014-1048-z

21. Morgenthaler TI, Aurora RN, Brown T, Zak R, Alessi C, Boehlecke B, Chesson AL Jr, Friedman L, Kapur V, Maganti R, Owens J, Pancer J, Swick TJ, Standards of Practice Committee of the A, American Academy of Sleep M (2008) Practice parameters for the use of autotitrating continuous positive airway pressure devices for titrating pressures and treating adult patients with obstructive sleep apnea syndrome: an update for 2007. An American Academy of Sleep Medicine report. Sleep 31(1):141-147

22. Massie CA, McArdle N, Hart RW, Schmidt-Nowara WW, Lankford A, Hudgel DW, Gordon N, Douglas NJ (2003) Comparison between automatic and fixed positive airway pressure therapy in the home. Am J Respir Crit Care Med 167(1):20-23. https://doi.org/10.1164/rccm.200201-022OC

23. Okuno K, Pliska BT, Hamoda M, Lowe AA, Almeida FR (2015) Prediction of oral appliance treatment outcomes in obstructive 
sleep apnea: a systematic review. Sleep Med Rev 30:25-33. https://doi.org/10.1016/j.smrv.2015.11.007

24. Camacho M, Riaz M, Tahoori A, Certal V, Kushida CA (2015) Mathematical equations to predict positive airway pressures for obstructive sleep apnea: a systematic review. Sleep disorders 2015:-293868. https://doi.org/10.1155/2015/293868

25. Dempsey JA, Veasey SC, Morgan BJ, O'Donnell CP (2010) Pathophysiology of sleep apnea. Physiol Rev 90(1):47-112. https://doi.org/10.1152/physrev.00043.2008

26. Eckert DJ, White DP, Jordan AS, Malhotra A, Wellman A (2013) Defining phenotypic causes of obstructive sleep apnea. Identification of novel therapeutic targets. Am J Respir Crit Care Med 188(8):996-1004. https://doi.org/10.1164/rccm.2013030448OC

27. Tsuiki S, Ito E, Isono S, Ryan CF, Komada Y, Matsuura M, Inoue Y (2013) Oropharyngeal crowding and obesity as predictors of oral appliance treatment response to moderate obstructive sleep apnea. Chest 144(2):558-563. https://doi.org/10.1378/chest.12-2609
28. Kastoer C, Dieltjens M, Oorts E, Hamans E, Braem MJ, Van de Heyning PH, Vanderveken OM (2016) The use of remotely controlled mandibular positioner as a predictive screening tool for mandibular advancement device therapy in patients with obstructive sleep apnea through singlenight progressive titration of the mandible: a systematic review. J Clin Sleep Med: JCSM : Off Publ Am Acad Sleep Med 12(10):1411-1421. https://doi.org/10.5664/jcsm.6202

29. Okuno K, Sasao Y, Nohara K, Sakai T, Pliska BT, Lowe AA, Ryan CF, Almeida FR (2016) Endoscopy evaluation to predict oral appliance outcomes in obstructive sleep apnoea. Eur Respir J 47(5): 1410-1419. https://doi.org/10.1183/13993003.01088-2015

30. Sawyer AM, Gooneratne NS, Marcus CL, Ofer D, Richards KC, Weaver TE (2011) A systematic review of CPAP adherence across age groups: clinical and empiric insights for developing CPAP adherence interventions. Sleep Med Rev 15(6):343-356. https://doi.org/10.1016/j.smrv.2011.01.003 